\title{
Retinoblastoma and prune belly syndrome: the first described association in literature
}

\author{
Rafat Turki', Ibrahim Alnawaiseh $\oplus^{1}$, Ewa Jasińska $\oplus^{2}$, Agata Pietras-Baczewska $\oplus^{2}$, \\ Mustafa Mehyar', Yacoub A. Yousef $\odot^{1}$, Robert Rejdak $\oplus^{2}$, Rashed Mustafa Nazzal $\odot^{3}$ \\ 'Ophthalmology Department, King Hussein Cancer Center, Amman, Jordan \\ ${ }^{2}$ Ophthalmology Department, Medical University of Lublin, Lublin, Poland \\ ${ }^{3}$ Shami Eye Center, Amman, Jordan
}

\begin{abstract}
BACKGROUND: Retinoblastoma is the most common primary intraocular tumor in children. Prune belly syndrome is a rare congenital disease affecting newborns. The combination of the two diseases has never been reported before. In this article, we present a case of a male infant diagnosed with prune belly syndrome (PBS) at birth.

CASE REPORT: Patient underwent many surgeries to manage the resulting consequences of PBS. At the age of 7 months, he presented with left eye leukocoria. He was examined and diagnosed with retinoblastoma. The eye was enucleated, and the diagnosis was confirmed histopathologically. This is the first case to be described in the literature of such an association to the best of our knowledge.
\end{abstract}

KEY WORDS: prune belly syndrome; retinoblastoma; Eagle-Barrett syndrome

Ophthalmol J 2020; Vol. 5, 143-145

\section{INTRODUCTION}

Retinoblastoma is the most common intraocular primary malignancy in children, with an estimated incidence of 1 in 15,000 to 1 in 18,000 live births. There is no racial or gender predisposition for the tumor. It can be unilateral, bilateral, or even trilateral; in the latter case, the pineal gland is featuring a tumor as well. The average age at diagnosis is 18 months; unilateral retinoblastomas are diagnosed at an older age than bilateral tumors [1]. The genetic bases for the tumor were identified early on. The retinoblastoma gene (RB1) was the first tumor suppressor gene cloned. It is a negative regulator of the cell cycle through its ability to bind the transcription factor E2F and repress transcription of genes required for the $S$ phase. The gene is located on chromosome 13q14 [2].

On the other hand, prune belly syndrome (PBS), which is also known as Eagle-Barrett syndrome, is a rare congenital disorder characterized by the triad of deficient abdominal musculature, cryptorchidism, and urinary tract abnormalities [3, 4]. Prune belly syndrome has an incidence of 3.6 to 3.8 per 100,000 live male births [5]. It has a wide range of presentations from being incompatible with life to an almost normal child $[6,7]$. The severity of renal dysplasia largely determines the prognosis of this disease [8]. While PBS mainly affects the genitourinary tract, including different degrees of renal dysplasia, hydronephrosis, enlarged bladder, and urethral obstruction, multisystem involvement has been frequently reported [9].

Indeed, various gastrointestinal, cardiac, pulmonary, skeletal manifestations have been associated with PBS, including pulmonary hypoplasia, patent ductus arteriosus, imperforated anus, club foot, and congenital hip dislocation [10]. Given the very low incidence of each disease alone, statistically 
speaking, the probability of both conditions to exist together is exceedingly rare. This article is by far the first case to be reported with such association. Whether this simultaneous occurrence is causative or by chance still to be elucidated.

\section{CASE REPORT}

The patient is a male, born from nonconsanguineous parents, and delivered vaginally. Antenatal ultrasound revealed oligohydramnios and a large fetal bladder. The neonate has prune-like abdominal skin wrinkles, bilateral cryptorchidism, and developmental hip dysplasia (Fig. 1). He was admitted to the neonatal intensive care unit for multiple urinary tract infections and underwent vesicostomy due to severe bilateral hydronephrosis, chronic kidney disease, and vesicoureteral reflux stage $\mathrm{V}$. By the age of 7 month, his mother noticed left eye leukocoria. Fundus examination of the left eye, performed under anesthesia, showed intraocular mass occupying the vitreous cavity (endophytic growth) with total exudative retinal detachment and subretinal seeds. The right eye was normal. B-scan ultrasound of the left eye showed numerous hyperechoic calcifications within the mass (Fig. 2). Magnetic resonance imaging (MRI) revealed a large left intraocular mass, measuring around $1.3 \times 1.3 \mathrm{~cm}$ on axial images and around $1.6 \mathrm{~cm}$ craniocaudally. There was an intermediate signal on the T1-weighted sequence, an intermediate to slightly hypointense signal on the T2-weighted sequence, and a deep hypointense T2 signal on the short T1-inversion recovery (STIR) sequence. The patient was diagnosed with left eye retinoblastoma group D and underwent left eye enucleation. Histopathologic assess-

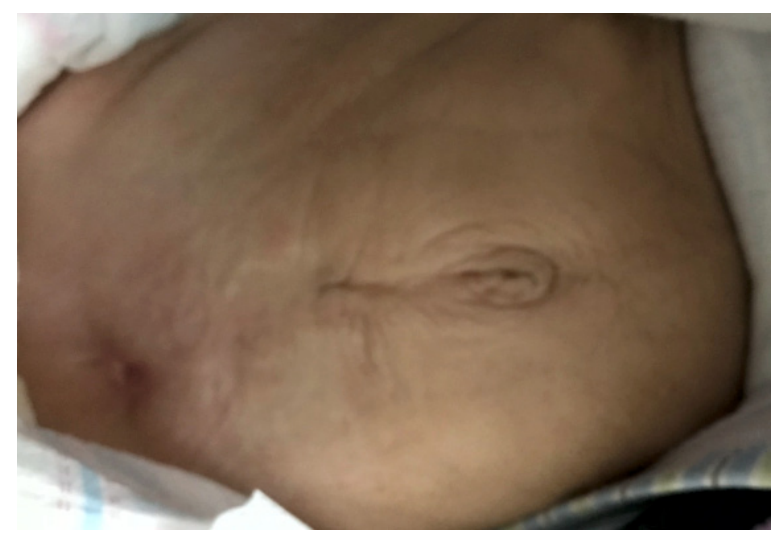

FIGURE 1. Abdominal image showing scar of previous surgeries to manage the urological consequences of the disease ment of the enucleated eye confirmed the diagnosis of retinoblastoma with massive choroidal invasion.

\section{DISCUSSION}

Both retinoblastoma and prune belly syndrome can take place as an isolated event or as a familial disease. In familial cases, both conditions could have a genetic background. Initially, a possible genetic basis for PBS was discounted mainly due to reports of monozygotic twins discordant. Nevertheless, few publications have suggested the possibility that this syndrome can have a genetic background when case reports of familial PBS primarily affecting brothers have suggested a possible autosomal or X-linked recessive mode of inheritance. Thus, a genetic basis for PBS is highly suggested [5].

Further studies have currently identified $H N F 1 \beta$ as the only candidate PBS gene based on two published PBS cases with chromosome $17 \mathrm{q} 12$ microdeletions encompassing the $H N F 1 \beta$ gene [11]. Although some authors have reported an ocular association to be present in some cases of PBS, these features are usually part of Potter's faces, which might be present secondary to oligo-hydramnios rather than being genuine associated features. These include: micrognathia, wide-set eyes, flattened palpebral fissures, prominent epicanthus, flattened nasal bridge, low-set ears lacking cartilage, and skeletal deformities [12].

Nevertheless, few reports described genuine associations: micro-cornea, primary optic atrophy, persistent pupillary membrane, and congenital mydriasis $[13,14]$. Although 13q deletion syndrome can sometimes be associated with retinoblastoma and urological problems, it has never been reported as a PBS cause. It is a rare genetic disease caused by

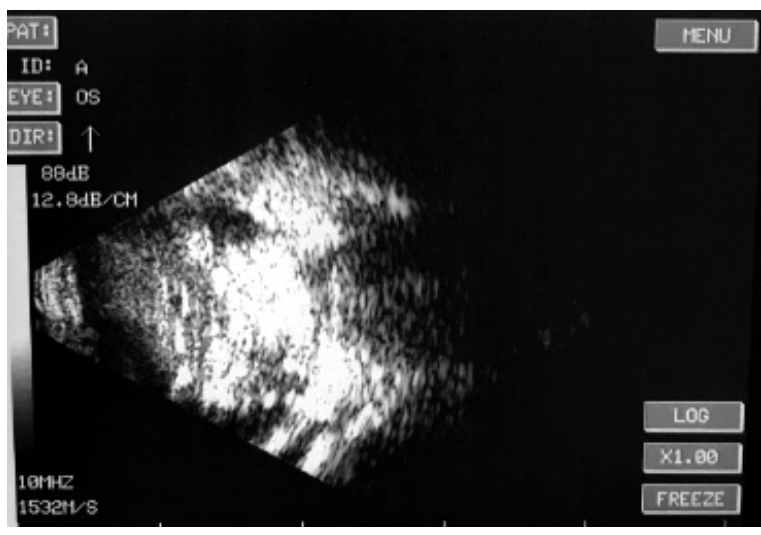

FIGURE 2. Ocular B-scan ultrasonography illustrating the intraocular mass with calcifications 
the deletion of some or the entire large arm of human chromosome 13. Different areas of deletion are associated with various symptoms, including: mental retardation, broad prominent nasal, bridge, hypertelorism, microphthalmia, epicanthus, ptosis, colobomata, retinoblastoma, micrognathia, protruding maxilla, low set ears, facial asymmetry, congenital heart disease, imperforate anus, Hypospadias or epispadias, undescended testes, bifid scrotum, pelvic girdle anomalies, and foot and toe anomalies [15].

\section{CONCLUSION}

Retinoblastoma and PBS have never been reported in the literature, and to the best of our knowledge, this is the first case report to register such an association. Whether this has a causative relationship or not is unclear.

\section{Acknowledgments}

None.

\section{Declaration of conflicting interests}

The authors declare that there is no conflict of interest.

\section{Ethics}

This article adheres to the World Medical Association Declaration of Helsinki.

\section{Consent}

The patient's written informed consent and images were provided by the patient's legally authorized representative (father).

\section{Funding}

None.

\section{REFERENCES}

1. Shields JA. Intraocular tumors - a text and atlas. WB Saunders Company, Philadelphia 1992.

2. Hanahan D, Weinberg R. The Hallmarks of Cancer. Cell. 2000; 100(1): $57-70$, doi: $10.1016 / \mathrm{s} 0092-8674(00) 81683-9$, indexed in Pubmed: 10647931.

3. Seidel NE, Arlen AM, Smith EA, et al. Clinical manifestations and management of prune-belly syndrome in a large contemporary pediatric population. Urology. 2015; 85(1): 211-215, doi: 10.1016/j. urology.2014.09.029, indexed in Pubmed: 25444629.

4. Eagle JF, Barrett GS. Congenital deficiency of abdominal musculature with associated genitourinary abnormalities: A syndrome. Report of 9 cases. Pediatrics. 1950; 6(5): 721-736, indexed in Pubmed: 14797335.

5. Granberg CF, Harrison SM, Dajusta D, et al. Genetic basis of prune belly syndrome: screening for HNF1 gene. J Urol. 2012; 187(1): 272-278, doi: 10.1016/j.juro.2011.09.036, indexed in Pubmed: 22114815.

6. Diao B, Diallo Y, Fall PA, et al. [Prune Belly syndrome: epidemiologic, clinic and therapeutic aspects]. Prog Urol. 2008; 18(7): 470-474, doi: 10.1016/j.purol.2008.04.003, indexed in Pubmed: 18602609.

7. Zugor V, Schott GE, Labanaris AP. The Prune Belly syndrome: urological aspects and long-term outcomes of a rare disease. Pediatr Rep. 2012; 4(2): e20, doi: 10.4081/pr.2012.e20, indexed in Pubmed: 22802998.

8. Hillman RT, Garabedian MJ, Wallerstein RJ. Pregnancy outcome in a woman with prune belly syndrome. BMJ Case Rep. 2012; 2012, doi: 10.1136/bcr-2012-006490, indexed in Pubmed: 23203170.

9. Sutherland RS, Mevorach RA, Kogan BA. The prune-belly syndrome: current insights. Pediatr Nephrol. 1995; 9(6): 770-778, doi: 10.1007/ BF00868740, indexed in Pubmed: 8747124.

10. Grimsby GM, Harrison SM, Granberg CF, et al. Impact and frequency of extra-genitourinary manifestations of prune belly syndrome. J Pediatr Urol. 2015; 11(5): 280.e1-280.e6, doi: 10.1016/j.jpurol.2015.06.005, indexed in Pubmed: 26231776.

11. Murray PJ, Thomas K, Mulgrew CJ, et al. Whole gene deletion of the hepatocyte nuclear factor-1beta gene in a patient with the prunebelly syndrome. Nephrol Dial Transplant. 2008; 23(7): 2412-2415, doi: 10.1093/ndt/gfn169, indexed in Pubmed: 18411231.

12. Woods AG, Brandon DH. Prune belly syndrome. A focused physical assessment. Adv Neonatal Care. 2007; 7(3): 132-43; quiz 144, doi: 10.1097/01.anc.0000278211.95767.05, indexed in Pubmed: 17844777.

13. Brodsky MC, Turan $\mathrm{KE}$, Khanna $\mathrm{CL}$, et al. Congenital mydriasis and prune belly syndrome in a child with an ACTA2 mutation. J AAPOS. 2014; 18(4): 393-395, doi: 10.1016/j.jaapos.2014.02.010, indexed in Pubmed: 24998021.

14. Shorey $P$, Lobo G. Ocular anomalies in abdominal muscle deficiency syndrome. Am J Ophthalmol. 1989; 108(2): 193-195, doi: 10.1016/0002-9394(89)90019-6, indexed in Pubmed: 2757102.

15. Grace E, Drennan J, Colver D, et al. The 13q- deletion syndrome. J Med Genet. 1971; 8(3): 351-357, doi: 10.1136/jmg.8.3.351, indexed in Pubmed: 5097142. 